\title{
Blending video games with learning: Issues and challenges with classroom implementations in the Turkish context
}

\section{Hakan Tüzün}

Hakan Tüzün is an Assistant Professor in the Department of Computer Education and Instructional Technology at Hacettepe University in Ankara, Turkey. His current work involves the design of rich learning environments through the use of new technologies and consideration for the culture of the learners and the communities they are a part of. More information about his work can be found at http://yunus.hacettepe.edu.tr/ htuzun. Address for correspondence: Dr Hakan Tüzün, Hacettepe Üniversitesi, Bilgisayar ve Öğretim Teknolojileri Eğitimi Bölümü (BÖTE), 06800, Beytepe/Ankara, Turkey.Tel: (90-312)2977176; email: htuzun@hacettepe.edu.tr

\begin{abstract}
The research design for this study focuses on examining the core issues and challenges when video games are used in the classroom. For this purpose three naturalistic contexts in Turkey were examined in which educational video games were used as the basis for teaching units on world continents and countries, first aid, and basic computer hardware and peripherals, in primary, secondary and higher education contexts respectively. Methods employed in the data collection include observing lessons, taking field notes, interviewing students and teachers, saving online discourse data, and collecting student artifacts and reflections. Findings identified issues related to (1) the design of the video game environment, (2) school infrastructure, (3) the nature of learning, the role of the teacher and classroom culture, and (4) engagement.
\end{abstract}

\section{Introduction}

The formal learning environment is surrounded with a culture of vision and mission, an interest of stakeholders, a framework of curriculum and other constraints. Although numerous studies have evaluated the impact of video games on achievement (eg, Corbit \& DeVarco, 2000; Cordova \& Lepper, 1996) and motivation (eg, Malone \& Lepper, 1987; Tüzün, 2006), research studies in this area have failed to consider the importance of the learning context. There are few video game studies (eg, EgenfeldtNielsen, 2004) that were designed around the constraints of the learning context, such as strategically aligning the learning tasks with the needs of the learners. Examining the utilisation of video games in real-world educational contexts under these constraints will likely inform teachers and instructional designers about how video 
games can be meaningfully and realistically blended with conventional learning environments.

\section{Related literature}

The younger generation is characterised as being different from the older generations. Frand (2000) identifies this generation with the following essential characteristics: computers and the Internet are a part of their life, staying connected through technology is essential, multitasking is natural and doing is more important than knowing. With these characteristics, it is not surprising that this generation does not respond to traditional teaching approaches such as lecturing, as Prensky (2000) argues. Advocates point out that video games match the characteristics of the younger generation, who grew up playing video games, and that they can be integrated into classroom learning to connect with this generation (eg, Gee, 2003).

According to Van Eck (2006), educators have followed three approaches for integrating video games into their classrooms: having educators or developers design games from scratch, having students design games from scratch and integrating commercial offthe-shelf (COTS) games into classroom learning. As Michael and Chen (2006) point out, the adoption of video games in the classroom largely depends on teachers and school administrators, who need to be convinced of their usefulness by being presented with concrete research showing that they do a better job than current teaching methods. There seems to be much propaganda about the use of video games for learning recently, and as Sefton-Green (2005, p. 419) emphasises, "the absence of empirical observations or audience studies or good industry-based research only helps the texts float free in this speculative ether.'

Much of the research related to video games and learning focused on achievement and motivation, used traditional methodologies like experimental designs, and was conducted in the US context. Although these studies have been valuable and show the significance of the medium for education, we need studies that go beyond and show its use in authentic classroom contexts that embrace more naturalistic design methodologies and examine the issue from an international perspective. Only after that can researchers show the true merit of the medium to teachers and administrators. This study is unique in that it tries to target three areas simultaneously. It contains three naturalistic studies in which educational video games were used as the basis for teaching units on world continents and countries, first aid and basic computer hardware and peripherals in primary, secondary and higher education contexts in Turkey respectively. The goal of this study is to examine the core issues and challenges of when video games are used in the classroom.

\section{Methodology}

Research design

This study is qualitative in nature and grounded on two sets of methodologies. The broader design of this study can be thought of as an example of design-based research. This methodology involves conducting design and research in the context of real-world 
settings. According to Brown (1992), 'classroom life is synergistic' (p. 141), ie, classroom aspects such as teachers, curriculum and assessment are interdependent, and these aspects form a systemic classroom integrity. Thus, one cannot study only one aspect of the classroom independent of other aspects, or change one aspect of it without affecting others. Design-based research is a methodological tool kit to introduce innovations into real-world classroom contexts, examine the impact of those innovations on learning, and derive evidence-based claims (Barab \& Squire, 2004).

Fishman, Marx, Blumenfeld, Krajcik and Soloway (2004) state that most design-based research projects do not consider the issues of scalability, sustainability and usability, and that 'this limitation must be overcome if research is to create usable knowledge that addresses the challenges confronting technology innovations when implemented in real-world school contexts' (p. 43). To overcome this limitation and also to increase external generalisability of the findings and claims, the narrower design of this study embraces three evaluative case studies. Yin (2003) adds that the analytic benefit of a study will be substantial when there are two or more cases in it. Descriptions and explanations of the three cases were provided in this study for a better understanding of the issues and challenges of blending video games with classroom instruction.

\section{Research contexts and participants}

Research context 1

This study was implemented between May 2 and May 16, 2005 in a private K-8 elementary school embedded in a state university in the centre part of Turkey. There were 1240 students between the ages of 7 and 14 . The average class size was 27 . The school had 144 teachers and 37 support personnel. There were three computer laboratories and each was equipped with 15 networked computers and a whiteboard. The school curriculum included Mathematics, Science, Social Studies, Turkish, English, Art, Music, Drama, Physical Education and Computers.

The school was part of a Comenius project under the EU Socrates Program. In this project, 17 schools from 15 countries have participated to learn about similarities and differences among the world's countries. This Comenius project required participating schools to carry out several tasks. The video game implementation at this school served as part of this Comenius project, specifically functioning as one of the on-site activities among local students. School administration had selected 24 students (12 girls and 12 boys) from the fourth and fifth grades based on their grade averages and willingness. These students voluntarily participated in the Comenius project activities after regular school hours. Almost all students had a computer at home and many of them played computer games. Social and economic statuses of the students' families were above average.

\section{Research context 2}

This study was implemented between May 27 and June 3, 2005 in a private K-12 school in the centre part of Turkey. There were 1200 students between the ages of 7 and 18 . The average class size was 24 . The school had 142 teachers and 38 support personnel. 
There was one computer laboratory equipped with 30 networked computers, a projector and a whiteboard. The secondary school curriculum included Mathematics, Geometry, Physics, Chemistry, Biology, Turkish Literature, English, French, Geography, History and Health Education.

Health Education is a 2-hour-per-week mandatory course in the Turkish education system to be taken at the ninth grade level. The video game implementation at this school served in delivering the course unit on first aid to a class of the ninth grade with 22 students (13 girls and 9 boys). All students had a computer at home. Social and economic statuses of the students' families were above average.

\section{Research context 3}

This study was implemented between March 4 and March 11, 2005 in a Computer Education and Instructional Technology (CEIT) department at a large state research university in the centre part of Turkey. In addition to providing courses to CEIT students, the department provided an introductory computer literacy course at undergraduate level to students of education faculty departments. There were 600 students between the ages of 19 and 22 taking this course in 12 sections. The average class size was 50. The department had nine academicians and nine support personnel. There were three computer laboratories in the department, and each was equipped with 15 networked computers and a whiteboard.

Computers is a 4-hour-per-week mandatory course to be taken by undergraduates in their sophomore year. The video game implementation at this school served to deliver the course unit on computer hardware and peripherals to a class of undergraduate math education students. There were 31 students (16 girls and 15 boys) in the class. About one third of the students had a computer at home. Social and economic statuses of the students' families were average.

Design of the video games

The Quest Atlantis (QA, Barab, Thomas, Dodge, Carteaux \& Tuzun, 2005) metagame platform was used for designing three separate video games to be used for three different subject matters and three different contexts. From a pedagogical perspective, the QA educational game is based on a framework that is inquiry-based and experiential (Dewey, 1938/1963). In addition, content in the game is situated as part of a rich narrative (Dickey, 2006). Learners are immersed into a 3D multiuser game environment in order to complete curricular activities, called Quests, as part of this narrative. Although these curricular activities are tailored towards 9- to 12-year-old students by design, the flexibly adaptive (Schwartz, Lin, Brophy \& Bransford, 1999) infrastructure of the game allows for scaling up of the game into contexts with differing needs.

A computer teacher from the elementary school context, a health education teacher from the secondary school context and an instructor from the university context were contacted conveniently about the potential utilisation of the QA game in their courses. These teachers approached the offer positively and obtained their administrations' 


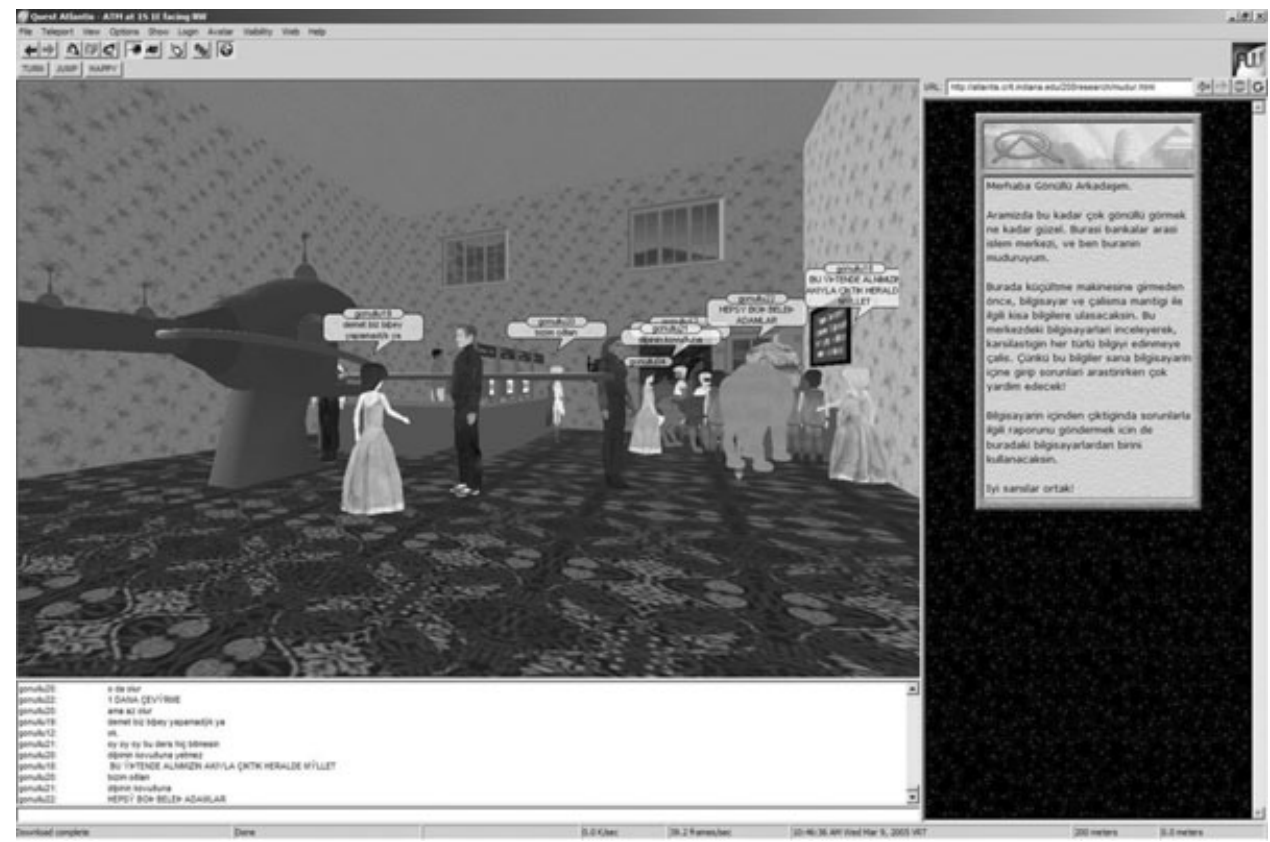

Figure 1: A screenshot from Virtual Computer game world, showing a scene while learners send their reports on why this computer did not function

authorisation to use the game in their classrooms. To fulfil the requirements for a graduate-level course, three teams of graduate students designed Countries, First Aid and Virtual Computer game worlds with their own unique narratives within the QA system under the guidance of the author. These game worlds were filled with structures, activities and curricular content based on the needs of three research contexts. In all implementations, students experienced a problem-based scenario. In the primary school context, the scenario involved non-playable characters (NPCs) that were lost in the game world. Students were given the task of finding out which countries these NPCs came from based on the information and clues provided in the environment. In the secondary school context, the scenario involved investigating themed areas in the firstaid world, like hospital and driver's licence course. In the university context, the scenario involved a non-functional giant computer. Students had to beam into this computer to figure out why it did not function based on the facts and information provided in the environment (Figure 1).

\section{Data sources}

Multiple methods of data collection were employed to enhance the validity and reliability of the study (Stake, 1995; Yin, 2003). In all three contexts the implementations were examined by observing lessons, taking field notes, interviewing students and teachers, saving online discourse data, and collecting student artifacts and reflections. 


\section{Data analysis}

Data analysis occurred in multiple stages as part of the ongoing data collection among the three research contexts so that successive data collection could be redirected (Stake, 1995). In categorising the issues and challenges of video game use in the classroom, the data sources were reviewed to develop a list of themes that seemed to be the most significant during the implementations and that would be most useful to others. This process resulted in the identification of four issues and related challenges.

\section{Findings}

Journal limitations prevent immersing readers into thick descriptions of the implementations. Therefore, common core issues and challenges in all three contexts when video games were used are presented in the following sections.

\section{Issue: design of the video game environment}

Because there was no available video game related to the subject matters needed by the three contexts, a design had to be undertaken for each. During the design, five challenges were faced. First, the design time was too excessive. The QA game environment uses a special client software that brings together a 3D virtual environment, a web browser and a communication window. Learners navigate in virtual 3D worlds to find learning tasks, use the web browser to collect data and submit their work, and use the discourse window to communicate with others in real time. Therefore, designing a new game world in QA means designing both for 3D and for the Web. Although construction in $3 \mathrm{D}$ is object-oriented and does not require programming, it still involves many tasks like finding or designing 3D objects, resizing and texturing them, and merging them in 3D space in a way similar to merging Lego pieces. For the content, there are special designer tools in QA for putting them into the Web; however, conceptualising inquirybased experiential activities precedes this process that requires much effort. On average, about 60 hours were spent for the development of each of the game environments.

Second, developing a relevant backstory accompanying the game environments was a challenging task. Even after development, students questioned the backstory and immediately found the logical faults instead of accepting the fantasy. For example, NPCs from different countries in the game implementation at the primary school context conveyed information in Turkish, but students questioned why these characters spoke in Turkish instead of their native languages. Third, it was observed that students compared the games with commercial ones, and had higher expectations from the game environments. For example, when the primary school students realised that they were not able to pick items they encountered, they were disappointed. Fourth, because QA environment has its own symbol systems that need to be mastered, students had to be oriented to the video game environment. The orientation included navigation in 3D, using first- and third-person perspectives, creating avatars, learning how to use the discourse window, discussing rules for social behaviour, using the resources in the Web window, opening the Quests and responding to Quests. For each case, a session at the beginning of the implementation had to be devoted for the orientation. And fifth, the design had to be conceptualised around time constraints of lessons. All of the contexts 
had limited time slots assigned for the chosen subject matters. This was three sessions for the primary and secondary schools and two sessions for the university. It was absolutely not possible to overflow these limitations because the contexts had to cover other subject matters in the remaining weeks according to their curriculum plans.

\section{Issue: school infrastructure}

The use of video games at schools required access to computers and the Internet. Because the schools did not have mobile technology like laptops, the classes had to be physically relocated from the classroom to the computer laboratory. This was instantly possible for the primary and secondary schools but not for the university. A computer laboratory was not available for the university classroom during its weekly scheduled class meeting. For that reason, another day and time had to be scheduled for this classroom based on the availability of the computer laboratories, the instructor and the students. Three additional challenges were faced related to infrastructure. First, the games had to be made available on the computers ahead of time before the implementation to prevent loss of time. Second, emergent technical problems were a part of implementation in authentic contexts. In the primary school context, the Internet connection was lost in the first week of the implementation, and supplementary paperbased activities had to be developed just in time to sustain the implementation. Similarly, a firewall at the secondary school context prevented the students' access to instructional content, and the content had to be made available on a local Intranet server just in time to sustain the implementation. At the university context, several computers crashed during implementation and they had to be restarted, which impacted the experience of students using these computers. And third, there was a lack of immediate technical support to solve these emergent technical problems in all three contexts. Researchers and developers were accompanying the implementations at the schools and they were fast in responding to these problems. Their absence could have hindered the efficient implementation of the games because they, as designers, knew the specifics of the games well and therefore were able to produce just-in-time solutions. An ordinary technical staff member could have been useless, because in most of the cases the solutions required interference to the design.

Issue: nature of learning, role of teacher and classroom culture

In parallel to problem-based scenarios, all students actively searched for information and clues in the game environments. Then they inquired into reasons for problems in the scenarios and took actions to solve them. For example, students at the primary school context interacted with NPCs to find out which countries they came from. They also searched for artifacts in the game environment that are dropped by these NPCs and tried to match them with the NPCs. Students at the secondary school context were charged with completing tasks related to electrical shocks, poisoning and injuries. To do so, they visited themed environments in the game world, interacted with NPCs and responded to questions in the environment. This created an experience for students in which they were navigating the game worlds and the content at their self-pace and at their control. In such a context, the role of the teacher shifted from an instructor to a guide. This did not mean, however, that the workload of the teacher was minimised and 
that the teacher became obsolete. Teachers floated among the students as helpers to follow their students' participation, and to take on many questions related to content, game experience and technical problems. At times when teachers needed help with these questions, researchers and designers supported them.

At the primary and secondary school contexts, each student was able to use a single computer. At the university context, two students had to be assigned to a single computer. Solving problems in the scenarios did not specifically ask for collaboration among students. However, in all contexts, even at the primary and secondary school contexts where a student had access to a single computer, a natural collaboration and help in solving game assignments and tasks emerged among students. Students at different workstations visited each other's computer, or they observed and cooperated with students next to them. In addition, students utilised the discourse window for collaboration. It was observed the volume of discourse transcripts increased as the levels increased from the primary school to the university. However, the nature of using the discourse function was more for instructional purposes among the primary school students and less among the secondary school and university students. The latter student group used this function more for leisure and socialising. All these collaboration activities created a positive, messy classroom culture, in which students were active participants. While teachers acknowledged this messy culture and repositioned their roles from an instructor to a guide from the content perspective, they were still at the centre of the classroom from the management perspective. Students still perceived teachers as the authority for management and responded to requests of the teachers in providing order in the classroom.

\section{Issue: engagement}

Students clearly enjoyed learning in these game-based learning environments. Because the design of the games required participation at different levels, like navigating to a certain area, collecting information, submitting work, etc, balancing these tasks for some students between entertainment and learning was a challenge. For example, some students in the primary school context spent much time in navigating and exploring structures in the virtual game world. Similarly, some students at the secondary school and university contexts spent much time at the beginning on chatting and lost their focus on learning tasks. In either case, the teachers had to direct these students' attention to learning tasks so that they could finish the whole experience in the limited time assigned for the class.

\section{Discussion and implications}

Van Eck (2006) identified integrating COTS games into classroom learning as the most suitable approach while acknowledging the limited topics and inaccurate or incomplete content COTS games may provide. This research supported this acknowledgement. In each of the three cases, teachers approached the video game implementation offer from content perspective and they demanded specific content and topics in the domains they taught. After an evaluation of the educational and COTS games, it was evident that none of the available titles targeted content in the needed topics, let alone content in 
Turkish. For that reason, a design endeavour had to be embraced for each of the learning contexts.

Some opponents of video game use in education (eg, Thornburg, 2006) criticise the decontextualised learning experience that some of the titles provide, such as the experience that can be found in drill and practice type of games, and argue that there are gaming environments of value if designed well. A good game design requires attention to many details. A problem-based approach provides a context for learning and is the dominant paradigm in the design of current game-based learning environments. This approach simply is not sufficient for a good game, and it needs to be supported with a well-thought scenario, and environments (usually 3D) with visually and functionally appealing structures in it. However, as shown by our experience and as supported by the literature (Allery, 2004), such a good game design is a time and resource consuming process. Even after spending that much effort, our designs are weighed by learners against professional products, which are backed up by a $\$ 7$ billion-per-year industry (Entertainment Software Association, 2006). Therefore, the most powerful aspect of video games for learning, providing engagement, could actually be one of the biggest barriers in using them in classroom settings, because this characteristic comes with a cost. Add to that barrier the rapid consumer characteristic of the younger generation, and we have an even bigger problem of providing sustainable video game environments for these learners. Sooner or later, models of funding for designing video games for education need to be found.

Many of the video game studies conducted in conventional learning environments treated the issue of time as expandable (eg, Egenfeldt-Nielsen, 2004). On the other hand, evident from this research, these environments still are surrounded by the "education as production' metaphor (Sawada \& Caley, 1985, p. 14), in which there is a well-established curriculum to 'process'. Courses in this study did not have the luxury of expanding into another hour because students would attend another course and teachers would teach another class, or into another week because teachers had to cover something else in the curriculum. Longer implementation times through computer games might decrease the buy-in among teachers, students, administrators and parents, and could be a barrier in rich technology implementations at schools in cultures like Turkey, where the educational system is shadowed by national school entrance exams.

Learners should have an opportunity for mastering the game in the form of an orientation; otherwise they will be frustrated and will lose their interest towards the game (White, 1984). This adds another layer to the issue of time. The implementations in this study took one of the available sessions for orientation. A systemic video game design embracing multiple disciplines, subject areas and units can eliminate the need for orientation for every instance of a game implementation in a classroom context.

Time alone is not sufficient for video game implementation in a school context and it should be supported by a reliable, strong and available information technology (IT) 
infrastructure. Most of the present video game implementations at schools require the use of muscular computers housed in a closed environment. In schools, computer laboratories are well suited for this purpose; however, availability seems to be an issue. With current student volumes, computer laboratories can barely be enough for teaching about basic IT skills. To go beyond, either more computer laboratories need to be added, or the computer technology needs to be brought into the classroom. Mobile computer technology has matured enough for economical use in the classroom. Therefore, schools need to change their vision for providing IT infrastructure to their students if they want to make video games a part of their curriculum. Availability is not the only issue. As new video game titles come out, they increase their demand for hardware; therefore, for many people, playing the latest video games is one of the main motives behind upgrading or totally replacing their computers every 2 years. This can be economically challenging for schools. One solution might be to come up with game designs that will tailor itself for both high-end and low-end computers. The available and adequate IT infrastructure should also be supported for a reliable usage. In this study, video game software had to be installed, configured and tested, and emergent technical problems had to be eliminated. Hardware, software and network support are the essential core elements for any technology implementation at schools (Tüzün \& Yilmaz, 2002). In addition to this core requirement, support personnel should be familiar with the particulars of a game design in order to solve emergent technical problems. This entails designers to document their games well. Overall, the availability, adequacy and reliability of IT infrastructure may be a burden for a successful video game implementation at schools as a result of limited budgets they have in general.

Schools might have sufficient time and an available, adequate and reliable IT infrastructure to implement a well-designed video game; nevertheless, teachers need to be aware of the 'cultural infrastructure' (Herz, 2002, p. 175) these resources will generate. As noted by Madden, Ford, Miller and Levy (2003), teachers usually utilise new technologies in the classroom in the way they used to teach, without changing their teaching methods or beliefs. As an example, a study conducted in 25 technology-rich primary and secondary school classrooms in five European countries found that Information and Communication Technology (ICT) in many cases was used to supplement traditional ways of teaching (Smeets \& Mooij, 2001). One of the reasons for this could be multiple affordances (Norman, 1988) provided by a type of technology. According to Dickey (2003), the affordances and constraints of a learning environment impact the opportunities for learning. In the video game implementations, being in control of one's own learning, active participation in learning activities and collaboration through discourse with peers emerged as the natural affordances of game-based learning environments. These are very much the characteristics of constructivist (Jonassen, 1999) and social constructivist (Vygotsky, 1933/1978) pedagogies. Because the affordances provided by video games seem to constrain teachers from following the traditional didactic teaching model, introduction of video games into the classroom might function as a Trojan horse to truly transform classroom learning. As a result, blending video games with classroom learning might facilitate the adoption of constructivist epistemology at classrooms, where the objectivist perspective is still at the centre today. 
Teachers are an important part of this cultural infrastructure, but through new roles. In the video game implementations, teachers emerged as a floating helper, dealing with technical problems and concept explanations, and guiding learners on doing tasks, an active role also defined as 'scaffolding'. Teachers' management skills in the classroom seemed to be a central part of their new role. This matches general teacher characteristics well because teachers have a tendency to be in control of the learning environment even when they act as coaches (Smeets \& Mooij, 2001). Overall, the adoption of this new role by teachers when video games are used in the classroom requires an orientation of use for teachers as well, because teachers usually have deficiencies when they utilise innovative ICT uses (Hinostroza, Guzman \& Isaacs, 2002). This orientation was provided to teachers on the fly by designers in this study, but scaleable video game implementations require more professional approaches to teacher training, such as in the form of a teacher professional development course.

Computer laboratory environments in schools need to be designed to facilitate the most productive approaches to learning (Brett \& Nagra, 2005). The physical arrangements of learning environments in this study contributed to the formation of a classroom culture in which collaboration activities emerged as a natural affordance. All the laboratories utilised had computers around the walls with an open space in the middle. This arrangement of equipment seemed to facilitate the natural collaboration and interaction among the learners, while the open space in the middle allowed the teachers to help and monitor their students. The implication of this finding is that learning environments at schools should be arranged in a similar fashion when using video games in their curriculum.

Video games are multimodal tools that include 3D spaces, conversational tools, audio, video, graphics, animation and AI (De Castell \& Jenson, 2004). In alignment with previous findings (eg, Tüzün, 2006), most of the students participated in the implementations in this study through a pattern in which the immersive context of the game faded away, and the learning opportunities and the social relations around them took precedence. A number of students though seemed to be totally absorbed in some of the modalities just for leisure. Teachers present in the learning environment emerged as an important factor to balance the participation of these students between playing and learning. This again points to the important role of the teacher as a manager of the classroom and is proof that teachers still have job security even when innovative approaches to learning are present in the classroom.

\section{Conclusion}

The research in this paper shows the potential of video games in traditional classroom settings and also provides grounded support for some of the game-based learning claims being repeated in the literature. It is evident that the issues and challenges of blending video games with classroom learning are multifaceted and interconnected. While the presented research exposed some of them, additional studies are needed to provide more depth to this multifaceted phenomenon. 


\section{Acknowledgements}

The author acknowledges the effort of graduate students, including Turgay Baş, Yasemin Demiraslan, Oktay Dönmez, Yavuz İnal, Türkan Karakuş, Gonca Kızılkaya and Meryem Yllmaz Soylu. Special thanks to Dr Arif Altun, Gregory C. De Sarro and Dr Özgül Yılmaz Tüzün for their valuable contributions through the reviews of initial manuscripts. The author is a member of QA R\&D team. QA is based upon work supported by the National Science Foundation under grant numbers 9980081, 0092831 and 0411846 .

\section{References}

Allery, L. A. (2004). Educational games and structured experiences. Media Teacher, 26, 6, 504505.

Barab, S. \& Squire, K. (2004). Design-based research: putting a stake in the ground. The Journal of the Learning Sciences, 13, 1, 1-14.

Barab, S., Thomas, M., Dodge, T., Carteaux, B. \& Tuzun, H. (2005). Making learning fun: Quest Atlantis, a game without guns. Educational Technology Research and Development, 53, 1, 86-107.

Brett, P. \& Nagra, J. (2005). An investigation into students' use of a computer-based social learning space: lessons for facilitating collaborative approaches to learning. British Journal of Educational Technology, 36, 2, 281-292.

Brown, A. L. (1992). Design experiments: theoretical and methodological challenges in creating complex interventions in classroom settings. The Journal of the Learning Sciences, 2, 2, 141178.

Corbit, M. \& DeVarco, B. (2000). Scicentr and bioLearn: two 3-D implementations of CVE science museums. In E. Churchill and M. Reddy (Eds), Proceedings of the Third International Conference on Collaborative Virtual Environments (pp. 65-71). New York: ACM.

Cordova, D. I. \& Lepper, M. R. (1996). Intrinsic motivation and the process of learning: beneficial effects of contextualization, personalization, and choice. Journal of Educational Psychology, 88, $4,715-730$.

De Castell, S. \& Jenson, J. (2004). Paying attention to attention: new economies for learning. Educational Theory, 54, 4, 381-397.

Dewey, J. (1938/1963). Experience and education. New York: Macmillan Publishing Company.

Dickey, M. D. (2003). Teaching in 3D: pedagogical affordances and constraints of 3D virtual worlds for synchronous distance learning. Distance Education, 24, 1, 105-121.

Dickey, M. D. (2006). Game design narrative for learning: appropriating adventure game design narrative devices and techniques for the design of interactive learning environments. Educational Technology Research and Development, 54, 3, 245-263.

Egenfeldt-Nielsen, S. (2004). Practical barriers in using educational computer games. On the Horizon, 12, 1, 18-21.

Entertainment Software Association (2006). Essential facts about the computer and video game industry [Brochure]. Retrieved October 29, 2006, from http://www.theesa.com/archives/files/ Essential\%20Facts\%202006.pdf

Fishman, B., Marx, R., Blumenfeld, P., Krajcik, J. S. \& Soloway, E. (2004). Creating a framework for research on systemic technology innovations. Journal of the Learning Sciences, 13, 1, $43-76$.

Frand, J. (2000). The information age mindset: changes in students and implications for higher education. Educause Review, 35, 5, 15-24.

Gee, J. P. (2003). What video games have to teach us about learning and literacy. New York: Palgrave/ Macmillan.

Herz, J. C. (2002). Gaming the system: what higher education can learn from multiplayer online worlds. In M. Devlin, R. Larson \& J. Meyerson (Eds), The Internet and the University: 2001 Forum (pp. 169-191). Boulder, CO: Educause. 
Hinostroza, J. E., Guzman, A. \& Isaacs, S. (2002). Innovative uses of ICT in Chilean schools. Journal of Computer Assisted Learning, 18, 4, 459-469.

Jonassen, D. (1999). Designing constructivist learning environments. In C. M. Reigeluth (Ed.), Instructional-design theories and models Vol. 2 (pp. 215-239). Mahwah, NJ: Lawrence-Erlbaum Associates.

Madden, A., Ford, N., Miller, D. \& Levy, P. (2003). Using the internet in teaching: the views of practitioners. British Journal of Educational Technology, 36, 2, 255-280.

Malone, T. W. \& Lepper, M. R. (1987). Making learning fun: a taxonomy of intrinsic motivations for learning. In R. E. Snow \& M. J. Farr (Eds), Aptitude, learning and instruction: conative and affective process analyses (pp. 223-253). Hillsdale, NJ: Lawrence-Erlbaum Associates.

Michael, D. \& Chen, S. (2006). Serious games: games that educate, train, and inform. Boston: Thomson Course Technology.

Norman, D. A. (1988). The psychology of everyday things. New York: Basic Books.

Prensky, M. (2000). Digital game-based learning. New York: McGraw-Hill.

Sawada, D. \& Caley, M. T. (1985). Dissipative structures: new metaphors for becoming in education. Educational Researcher, 14, 3, 13-19.

Schwartz, D. L., Lin, X., Brophy, S., \& Bransford, J. D. (1999). Toward the development of flexibly adaptive instructional designs. In C. M. Reigeluth (Ed.), Instructional-design theories and models Vol. 2 (pp. 183-214). Mahwah, NJ: Lawrence-Erlbaum Associates.

Sefton-Green, J. (2005). Changing the rules? Computer games, theory, learning, and play. Discourse: Studies in the Cultural Politics of Education, 26, 3, 411-419.

Smeets, E. \& Mooij, T. (2001). Pupil-centred learning, ICT, and teacher behaviour: observations in educational practice. British Journal of Educational Technology, 32, 4, 403-417.

Stake, R. (1995). The art of case study research. Thousand Oaks, CA: Sage.

Thornburg, D. (2006). Can games be used to teach? Learning and Leading with Technology, 33, 7, $6-7$.

Tüzün, H. (2006). Multiple motivations framework. In M. Pivec (Ed.), Affective and emotional aspects of human-computer interaction: game-based and innovative learning approaches (pp. 59-92). Amsterdam, The Netherlands: IOS Press.

Tüzün, H. \& Yilmaz, O. (2002). Instructor support in web-based instruction. Proceedings of World Conference on Educational Multimedia, Hypermedia and Telecommunications 2002(1), 19871992. Retrieved October 29, 2006, from http://dl.aace.org/10496

Van Eck, R. (2006). Digital game-based learning: it's not just the digital natives who are restless. Educause Review, 41, 2, 16-30.

Vygotsky, L. (1933/1978). Mind in society: the development of higher psychological processes. Cambridge, MA: Harvard University Press.

White, B. Y. (1984). Designing computer games to help physics students understand Newton's laws of motion. Cognition and Instruction, 1, 1, 69-108.

Yin, R. K. (2003). Case study research: design and methods (3rd ed.). Thousand Oaks, CA: Sage. 